\title{
Turning an advantage into a disadvantage: Ambiguity effects in lexical decision versus reading tasks
}

\author{
C. DARREN PIERCEY and STEVE JOORDENS \\ University of Toronto, Scarborough, Ontario, Canada
}

\begin{abstract}
When performing a lexical decision task, participants can correctly categorize letter strings as words faster if they have multiple meanings (i.e., ambiguous words) than if they have one meaning (i.e., unambiguous words). In contrast, when reading connected text, participants tend to fixate longer on ambiguous words than on unambiguous words. Why are ambiguous words at an advantage in one word recognition task, and at a disadvantage in another? These disparate results can be reconciled if it is assumed that ambiguous words are relatively fast to reach a semantic-blend state sufficient for supporting lexical decisions, but then slow to escape the blend when the task requires a specific meaning be retrieved. We report several experiments that support this possibility.
\end{abstract}

It is rare for some variable to have opposite effects across two tasks that ought to reflect similar underlying processes. For example, in the lexical decision task, strings of letters are presented one at a time, and participants are instructed to decide as quickly and as accurately as possible whether or not each string constitutes a correctly spelled English word. One would expect that this task would reflect basic word recognition processes, the same processes one uses when reading connected text. Thus, it would be reasonable to assume that variables that have a certain effect on lexical decision performance would have a qualitatively similar effect on reading tasks.

However, the effects of semantic ambiguity clearly do not follow this expectation. Lexical ambiguity refers to the number of meanings a word has. A word that has two or more meanings is called ambiguous (e.g., BAT), whereas a word that has a single meaning is called unambiguous (e.g., PIANO). The type of effect this variable produces is largely dependent on the task used to measure it.

In the lexical decision task, ambiguous words provide a performance advantage over unambiguous words. The critical dependent measures in the lexical decision task are the reaction time and accuracy with which items are categorized as words. Specifically, then, participants can categorize items as words faster and more accurately if they are ambiguous words than if they are unambiguous words. This ambiguity advantage was originally reported by Jastrzembski and Stanners (1975; Jastrzembski, 1981).

This research was supported by a National Sciences and Engineering Research Council of Canada operating grant awarded to S.J. We thank Tom Spalek for helpful comments and discussions, and Mike Masson, Jay Ruekl, Keith Rayner, Ron Borowsky, and especially Morton Ann Gernsbacher, for a very helpful review process. Correspondence should be addressed to C. D. Piercey, Department of Psychology, University of Alberta, P-220 Biological Sciences Building, Edmonton, AB, T6G 2E9 Canada (e-mail: dpiercey@psych.ualberta.ca).
Subsequent studies by Gernsbacher (1984) and by Millis and Buttons (1989) suggested that the ambiguity advantage might not be robust and that it was potentially due to variables other than number of meanings, which may have differed across the ambiguous versus unambiguous words. While stimulus confounds are always a possibility, studies that have controlled for the major variables known to affect lexical decision latencies have still shown an advantage for ambiguous over unambiguous words (Borowsky \& Masson, 1996; Kellas, Ferraro, \& Simpson, 1988). Thus, it appears safe to say that it is easier to decide that an item is a word if it is a word with several meanings rather than one with a single meaning.

The interesting dissociation we wish to focus on in the present paper is that lexical ambiguity has precisely the opposite effect when participants are instructed to read connected text for comprehension. The usual dependent measure in reading studies is the amount of time that participants fixate on critical words in the text. A number of investigators have shown that participants tend to fixate on ambiguous words longer than they fixate on unambiguous words (Duffy, Morris, \& Rayner, 1988; Rayner \& Duffy, 1986, 1987; Rayner \& Frazier, 1989). This ambiguity disadvantage is observed in a number of different empirical situations, but perhaps the most relevant is the condition where the disambiguating context does not come until after the ambiguous word has been fixated. In this condition, where participants experience the word without any disambiguating prior context, they still fixate ambiguous words longer than unambiguous words (Duffy et al., 1988). Thus, when reading connected text, participants take longer to process a word with multiple meanings than a word with only a single meaning.

In fact, the above description of ambiguity effects on reading performance is actually an oversimplification of a complex set of results. We will address these complexities in some detail after presenting our "efficient then in- 
efficient" explanation of the paradoxical results across the lexical decision and reading tasks. For present purposes, the theory is best considered and explained in light of an ambiguity advantage in lexical decision set in juxtaposition to an ambiguity disadvantage in reading performance.

The obvious question, then, is why the effects of semantic ambiguity differ across the lexical decision task and the reading task. In the remainder of the introduction, we first present a possible explanation for these disparate results and then describe how recent notions about the dynamic nature of lexical decision performance can be used to explicitly test this explanation.

\section{The "Efficient Then Inefficient" Explanation for the Differing Effects of Ambiguity}

The "efficient then inefficient" explanation we provide is inspired by commentaries to a paper by Joordens and Besner (1994). Joordens and Besner tested whether a distributed model of memory (Masson, 1991) could account for the ambiguity advantage observed on the lexical decision task. They found that although the model was capable of producing an ambiguity effect, it had great difficulties settling into one of the semantic activation patterns associated with an ambiguous word. Instead, it usually settled into a pattern that was a "blend" of the two learned meanings. Given the claim by Masson that lexical decision could be modeled by counting the number of cycles to settle into a correct semantic pattern, Joordens and Besner took their results as suggesting that the model had difficulties making correct lexical decisions to ambiguous items.

However, in commentaries to that paper by Masson and Borowsky (1995) and Rueckl (1995), and in a countercommentary by Besner and Joordens (1995), the possibility was discussed that perhaps correct lexical decisions could be made by the model even when it settled into a blend. The general notion is that although a blend is not exactly the same as any learned pattern, it is similar to two learned patterns. Lexical decisions could be founded on a familiarity assessment based on the similarity between the current pattern, or representation, that is being developed and patterns in memory. Thus, even when the pattern is a blend, it might be possible to correctly classify an item as a word. Moreover, as suggested by the results of Joordens and Besner (1994), the model might be able to classify ambiguous words faster than unambiguous words, because it gets to the blend state relatively quickly when presented with an ambiguous word. This possibility was subsequently confirmed in modeling work performed by Borowsky and Masson (1996).

The critical idea we want to take from all this is that it may be reasonable to think of an ambiguous word as being processed in two consecutive stages. First, there may be a relatively fast and efficient initial stage where the semantic activation pattern being activated for the ambiguous word moves toward a pattern that is a blend of its two meanings. After that, and only if need be, there would be a slower and less efficient second stage where the semantic activation pattern moves out of the blend pattern and toward one of the specific meaning patterns.

The different results of ambiguity observed across the lexical decision and reading tasks can be explained within this framework if the following assumptions are made. First, we assume that lexical decisions can be emitted at some point prior to complete semantic processing. As discussed below, there is a large body of evidence suggesting this is the case (e.g., Joordens \& Becker, 1997). Second, we assume that in the typical way the lexical decision task is performed, ambiguous words are able to reach the level of semantic activation pattern necessary for making a correct lexical decision faster than an unambiguous word can. This assumption is consistent with the simulation results of Joordens and Besner (1994) and Borowsky and Masson (1996). Third, we assume that the word reading task requires a more complete semantic activation pattern. That is, a blend state may be an insufficient place to finish processing an item when the task requires participants to read for meaning as opposed to simply deciding whether the item is or is not a word. Finally, we assume that the time it takes to get to a semantic pattern sufficient for supporting reading performance is longer and more error prone for an ambiguous word than for an unambiguous word due to difficulties escaping the blend state.

With this framework presented, we can now address the complexity of the reading literature alluded to earlier. The complexities are tied to considerations about how strong the different meanings of an ambiguous word are. A crude distinction can be drawn between two classes of ambiguous items: balanced ambiguous words, which possess two equally dominant meanings, and unbalanced ambiguous words, which have one dominant meaning and a second subordinate meaning. Given this distinction, the more complex description of ambiguity effects in reading can be summarized as follows. When no biasing context is provided prior to the presentation of the critical target word, balanced ambiguous words are fixated longer than unambiguous words, but unbalanced ambiguous words are not. However, when a biasing context is provided prior to the critical target word and that context supports the subordinate meaning of an ambiguous word, balanced ambiguous words are not fixated longer than unambiguous words, but now unbalanced ambiguous words are fixated longer than unambiguous words.

In fact, this more complicated set of results conforms nicely with the "efficient then inefficient" framework. In simulation work, Joordens and Besner (1994) have shown that as ambiguous words are made less balanced, their tendency to settle into a blend decreases, and they are processed more like unambiguous words. Thus, it makes sense that without a biasing context, only balanced ambiguous words would show a measurable disadvantage relative to unambiguous words. However, if a biasing context favorable to a subordinate meaning is introduced, that context would increase the likelihood of an unbalanced ambiguous word settling into a blend, and it would decrease the 
likelihood of a balanced ambiguous word settling into a blend. Thus, the observed results are exactly what we would expect if the ambiguity disadvantage observed in the reading literature were due to difficulties escaping a semantic blend state.

Our explanation of the processing of ambiguous words also fits nicely with the literature on priming effects with ambiguous items. For example, Marcel (1980) showed that early in processing, ambiguous words prime items related to either of their two meanings. However, if processing is allowed to continue, and if a biasing context is provided, priming effects will eventually occur only for items related to the meaning of the word that is consistent with the current context (see also Onifer \& Swinney, 1981; Seidenberg, Tanenhaus, Leiman, \& Bienkowski, 1982; Swinney, 1979). Thus, like the above explanation, the general idea is that the semantic activation pattern of ambiguous words first moves to some pattern that is "close" to all of that word's meanings (e.g., a blend), and then it shifts into a specific meaning pattern. ${ }^{1}$

Our general claim, then, is that relative to an unambiguous word, the semantic processing of an ambiguous word is initially fast as it moves toward a blend pattern, but then disproportionately slow as it moves out of the blend and toward a specific learned meaning. If a task can be performed on the basis of a partially formed semantic activation pattern, an ambiguity advantage will be observed. However, if the task requires a more complete semantic activation pattern-something more than a blend-an ambiguity disadvantage will be observed.

\section{Present Study}

The goal of the present study is to test our explanation of the differing effects of ambiguity as presented in the previous section. Our strategy is to vary the amount of semantic processing required prior to making a lexical decision. If we can increase the level of semantic activation pattern required for making a lexical decision beyond that required for a typical lexical decision task, we might see the ambiguity advantage begin to become a disadvantage. Demonstration of both an advantage and a disadvantage within the same task by varying only the required degree of semantic processing would provide strong support for the theory described above.

How does one vary the degree of semantic processing required for making a lexical decision? A number of studies now suggest that one way to do this is to manipulate the kind of nonwords that are presented. Basically, the more "wordlike" the nonwords are, the longer it takes participants to decide that the words are words, and the greater the semantic influences on those word decisions. For example, James (1975) demonstrated that concreteness effects are larger when the nonwords are more wordlike. Stone and Van Orden (1993) demonstrated that word frequency effects increase as the nonwords are made more wordlike. Joordens and Hockley (in press) replicated the Stone and Van Orden result and also showed that recognition memory is better for items presented in the context of more wordlike nonwords. Finally, Joordens and Becker (1997) demonstrated that semantic priming effects get larger and last over longer lags as the nonwords are made more wordlike. Taken together, these results clearly suggest that words presented in a lexical decision task are processed more deeply when the nonwords are more wordlike.

Thus, by systematically manipulating the "wordlikeness" of the nonword foils, we should be able to vary the degree of semantic processing that occurs prior to a lexical decision response (see Joordens \& Becker, 1997, for a detailed explanation of this issue). If the "efficient then inefficient" explanation is correct, we may see the ambiguity advantage typically observed in lexical decision performance change to a disadvantage in versions of the lexical decision task that require increased levels of semantic processing.

\section{EXPERIMENT 1}

The goal of Experiment 1 was straightforward. We simply wished to examine the ambiguity effect in lexical decision across a variety of nonword conditions lying on a "wordlikeness" continuum. Previous experiments examining the ambiguity effect have tended to use pronounceable nonwords (e.g., CHROG). In addition to the pronounceable condition, we examined a scrambled nonword condition in which the nonwords are even less wordlike (e.g., RHGCO) and a pseudohomophone condition in which the nonwords were more wordlike in the sense that they have a word's pronunciation (e.g., PHROG).

It is important to note that we are not the first to examine the ambiguity effect under different nonword conditions. Borowsky and Masson (1996) examined the ambiguity effect in two situations. In one condition the nonwords contained no vowels at all, so-called illegal nonwords (e.g., HKLRS). In the other, pronounceable nonwords were used. They found that an ambiguity advantage was observed only in the pronounceable condition. This is not overly surprising since word responses were very fast in the illegal nonword condition and were likely reflecting a discrimination of words and nonwords on the basis of purely orthographic information. Thus, the finding really just suggests that some semantic processing is required to produce an ambiguity effect.

However, there is one very nice aspect of the result observed by Borowsky and Masson (1996). The finding of no ambiguity advantage when illegal nonwords were used (or when a naming task was used) weakens the possibility that when an ambiguity effect is observed with their stimuli, it is due to some confounding variable across the ambiguous and unambiguous items. Given this, we chose to use their set of ambiguous and unambiguous items in our experiments.

Our primary interest here is not in the comparison of the scrambled to the pronounceable condition. In fact, we would expect those conditions to largely parallel the results of Borowsky and Masson (1996). Rather, our interest is in what happens when the nonwords are changed from 
Table 1

Reaction Times (RTs, in Milliseconds) and Percentage Error Rates

for Words Across the Three Nonword Conditions of

Experiment 1 and the Single Condition of Experiment 2

\begin{tabular}{|c|c|c|c|c|c|c|c|}
\hline \multirow[b]{2}{*}{ Measure } & \multicolumn{2}{|c|}{ Unambiguous } & \multicolumn{2}{|c|}{ Ambiguous } & \multirow[b]{2}{*}{ Difference } & \multicolumn{2}{|c|}{ Nonwords } \\
\hline & $M$ & $S D$ & $M$ & $S D$ & & $M$ & $S D$ \\
\hline \multicolumn{8}{|l|}{ Reaction time } \\
\hline Scrambled & 550 & 95 & 542 & 90 & 8 & 571 & 90 \\
\hline Pronounceable & 644 & 100 & 628 & 109 & $16^{*}$ & 724 & 110 \\
\hline Pseudohomophone & 687 & 118 & 676 & 103 & 11 & 811 & 164 \\
\hline Pseudo+ (Exp 2) & 799 & 280 & 786 & 284 & 13 & 832 & 291 \\
\hline \multicolumn{8}{|l|}{ Percentage error rate } \\
\hline Scrambled & 6.92 & 4.03 & 5.33 & 2.27 & 1.58 & 2.54 & 1.63 \\
\hline Pronounceable & 6.33 & 3.08 & 3.75 & 2.80 & $2.58^{\dagger}$ & 2.51 & 2.50 \\
\hline Pseudohomophone & 10.58 & 6.07 & 3.42 & 3.90 & $7.16^{\dagger}$ & 8.40 & 5.86 \\
\hline Pseudo+ $(\operatorname{Exp} 2)$ & 12.37 & 5.59 & 9.87 & 7.80 & $2.50^{\dagger}$ & 25.28 & 3.69 \\
\hline
\end{tabular}

pronounceable to pseudohomophones. That is, when the semantic activation pattern required for making a lexical decision is increased beyond that of previous studies examining the ambiguity effect in lexical decision, what happens to the ambiguity advantage? Does it reduce or reverse, as the "efficient then inefficient" explanation would suggest? Or does it get larger in the way that other semantic influences get larger as nonwords are made more wordlike?

\section{Method}

Participants. Thirty-six undergraduates from the University of Toronto at Scarborough participated in the experiment, 12 in each of the three nonword conditions. The experiment took approximately $20 \mathrm{~min}$ and participants received bonus credit toward their introductory psychology class. All participants had normal or corrected vision. Because the University of Toronto at Scarborough is extremely multicultural, it is not easy to get a participant group of native English speakers. However, most are proficient in English despite not having it as a first language. In this experiment, we accepted any participant who obtained an accuracy level greater than $80 \%$ on the task as being sufficiently proficient in English.

Procedure. The study utilized a running lexical decision task composed of a single block of 256 trials. Each trial consisted of the following sequence of events: (1) a 500-msec blank screen, (2) a 500msec presentation of a warning asterisk, (3) a 500-msec blank field, and (4) a letter string presented at the center of the screen until a response was emitted. Participants were instructed to decide whether the letter string did or did not constitute a correctly spelled English word. They were instructed to make their responses as quickly and accurately as possible. The next trial was automatically initiated upon response.

Apparatus and Materials. Testing was carried out on an IBMcompatible computer equipped with a 15 -in. SVGA color monitor. Participants used a MEL response box to make word and nonword decisions. One button on the box was labeled "word," the other "nonword." Programming was done in MEL Professional 2.0. The participants sat about $50 \mathrm{~cm}$ from the monitor.

The word stimuli used in the present experiment were the 64 pairs of ambiguous and unambiguous words used by Borowsky and Masson (1996). These two sets of words were matched on a num- ber of dimensions, including length and word frequency. The items themselves, as well as specific information with respect to the various dimensions on which they are controlled, can be found in the Borowsky and Masson article.

The nonwords used in this experiment were 128 triples of nonwords that were a slightly expanded version of the stimuli used by Joordens and Becker (1997). The triples were created in the following manner. First, a pseudohomophone was generated (e.g., PHROG). Then the initial letter of the pseudohomophone was changed in a manner that turned the item into a pronounceable nonword (e.g., CHROG). Finally, a scrambled version of the nonword was created by reordering the letters of the pronounceable nonword in a manner that made the item nonpronounceable (e.g., RHGCO). Depending on group membership, a specific participant saw only the pseudohomophone, pronounceable, or scrambled version of each nonword.

\section{Results}

The results from Experiment 1 are depicted in Table 1. Statistical analyses were performed on both the reaction time and error rate data. In both cases, the analyses consisted of a $2 \times 3$ mixed analysis of variance (ANOVA; ambiguity $\times$ nonword type) followed by pairwise $t$ tests examining the ambiguity effect in each nonword condition. ${ }^{2}$

With regard to the reaction time data, the ANOVA revealed a significant main effect of nonword type $\left[F(2,33)=5.62, M S_{\mathrm{e}}=20,209, p<.01\right]$. Specifically, in accord with previous findings, the time to correctly categorize words progressively increased as the nonwords were made more wordlike. The main effect of ambiguity approached significance but failed to reach it $[F(1,33)=$ 2.30, $\left.M S_{\mathrm{e}}=965, p=.14\right]$. The interaction between ambiguity and nonword condition was not significant $(F<1$, $p=.91$ ). Despite the nonsignificant main effect of ambiguity, planned $t$ tests examining the ambiguity effect in each nonword condition revealed that the ambiguity effect just failed to reach the conventional .05 alpha level in the pronounceable condition $[t(11)=2.13, p=.057]$, but was less than 1 in the other two conditions. Thus, the re- 
action time data suggest that although the means were in a direction consistent with an ambiguity effect, the effect in reaction times was small and seemingly unaffected by the nonword condition.

However, a significant main effect of ambiguity was obtained in the error data $\left[F(1,33)=21.58, M S_{\mathrm{e}}=.0012\right.$, $p<.001]$, and that ambiguity effect did interact with nonword type $\left[F(2,33)=4.47, M S_{\mathrm{e}}=.0012, p<.05\right]$. This interaction is due to the fact that the ambiguity effect on errors became progressively larger across the scrambled, pronounceable, and pseudohomophone conditions. Pairwise $t$ tests examining the ambiguity effect in each condition revealed that while the $1.58 \%$ effect observed in the scrambled condition was not significant $[t(11)=1.32$, $p=.21$ ], both the $2.58 \%$ effect observed in the pronounceable condition and the $7.17 \%$ effect observed in the pseudohomophone condition were significant $[t(11)=$ $2.47, p<.05$, and $t(11) 3.88, p<.01]$, respectively. Thus, the error rate data suggest that the ambiguity effect is affected by the nonword manipulation such that a progressively larger ambiguity effect is observed as the nonwords are made more wordlike.

\section{Discussion}

It is not completely clear why we observe the ambiguity effect primarily in the error rate data, whereas other investigators tend to observe it primarily in the reaction time data. However, the fact that we do observe the effects primarily in the error data does not compromise the interpretations of our findings in any manner. If some variable such as lexical ambiguity causes a link in the processing to be more or less efficient, this difference in efficiency could show through in reaction times, errors, or both. Whether it shows through in reaction times or error rates is most likely due to the strategy participants use when going from the results of processing to the emission of a response. For example, in some contexts, participants may try to respond after some time period, and may begin to do so regardless of whether the processing has been sufficient to support a response. In such a situation, more errors will be made to the less efficient stimulus class because it will be the furthest from being ready at the time the participant begins to respond. Note that this could occur in the absence of any difference in response time. However, if the participants waited until the processing was sufficient to support an accurate response before beginning to respond, we would expect little difference in error rates, but a difference in reaction time favoring the more efficient stimulus class. Obviously, participants could also use some strategy falling between these two, resulting in both faster responses and fewer errors to the more efficient stimulus class.

Given this, it may be relevant that for many of our participants, English was not their first language. Despite the fact that all of these participants were proficient in English, it may be the case that, for some reason, they are more likely to use the strategy that results in effects showing through in errors. It is not immediately obvious why this would be the case, but the results of this study are not an isolated case; we have observed a tendency for results to show through in errors in other research we have conducted with this participant pool (e.g., Joordens \& Hockley, in press; Joordens \& Piercey, 1996).

The important point in all this is that it is perfectly reasonable to compare our findings to those from studies showing reaction time effects, and to draw our interpretations accordingly. To this end, the finding of an increased ambiguity effect across the nonword conditions in the present experiment is consistent with other studies showing an increase in semantic influences as nonwords are made more wordlike (e.g., James, 1975; Joordens \& Becker, 1997; Joordens \& Hockley, in press; Stone \& Van Orden, 1993). Thus, a straightforward explanation of our results is that the ambiguity advantage has a semantic locus, and as semantic processing is increased, the ambiguity advantage increases as well.

In addition, the results provide a replication of the findings of Borowsky and Masson (1996). Recall that they found an ambiguity effect when pronounceable nonwords were used, but not when illegal nonwords were used. Similarly, we found an ambiguity advantage when pronounceable nonwords were used but not when scrambled nonwords were used. Thus, it appears as though some semantic processing is required for an ambiguity effect to be observed in lexical decision. This again suggests that when an ambiguity effect is observed with this stimulus set, it is probably not due to any confound of the ambiguous versus unambiguous items at an orthographic level.

Critically, however, the findings do not confirm our "efficient then inefficient" explanation of the different ambiguity effects across the lexical decision and reading tasks. The hope was that if we increased the semantic processing required prior to making a lexical decision, the ambiguity advantage would diminish or perhaps even turn to a disadvantage. Quite opposite to this, the ambiguity advantage increased.

Although the "efficient then inefficient" explanation was not confirmed, neither was it denied. The findings can easily be explained within that framework simply by arguing that the use of pseudohomophones was insufficient to force semantic processing to the "second stage" for ambiguous words. That is, we may simply have traversed further along the first stage of processing toward the blend state. Given that this portion of processing is presumed to be disproportionately fast for ambiguous words, the more of that processing we allow, the larger the observed advantage.

\section{EXPERIMENT 2}

Given the failure of Experiment 1 to either confirm or deny our explanation of the differing ambiguity effects across the lexical decision and reading tasks, Experiment 2 was an attempt to increase semantic processing further still. Specifically, on the basis of the referent 
model of lexical decisions described by Joordens and Piercey (1996), Joordens and Becker (1997) showed that word decisions could be made even longer, and semantic influences even stronger, when a number of additional modifications were made to the lexical decision task. Those modifications included increasing the proportion of nonwords relative to words, and preexposing the nonwords in a naming context to make them more familiar when they occurred in the lexical decision task.

Thus, in the present experiment, we attempted to increase semantic influences beyond the pseudohomophonic condition of Experiment 1 by modifying the procedure for that condition so as to increase the nonword-to-word ratio and to preexpose the pseudohomophones prior to the lexical decision phase. If these modifications increase semantic processing, and if the "efficient then inefficient" explanation is correct, perhaps we will see the ambiguity advantage observed in the pseudohomophone condition of Experiment 1 reduce in magnitude or reverse altogether.

\section{Method}

Participants. Fifteen undergraduates from the University of Toronto at Scarborough participated in the experiment in exchange for bonus credit toward their introductory psychology class. All participants had normal or corrected vision. In this experiment we accepted any participant who obtained an accuracy level greater than $70 \%$ on the task as being sufficiently proficient in English. The cutoff level was lower in this experiment than in Experiment 1 because the task is more difficult and we would expect a lower level of accuracy. The mean accuracy across all participants for this experiment was $73 \%$.

Procedure. The procedure was identical to that described in Experiment 1 except for the following three changes. First, only pseudohomophones were used as nonword stimuli in the present experiment. Second, an additional 64 pseudohomophones were generated and presented, bringing the total number of trials to 320 and increasing the nonword-to-word ratio from $1: 1$ to $3: 2$.

Finally, all 192 pseudohomophones were presented one at a time in a phase prior to the actual lexical decision experiment. The procedure for this phase simply involved presenting a 500-msec blank field followed by a nonword. The nonword stayed on the screen until the participant read it aloud, at which time it disappeared and the next trial was initiated. No data were recorded during this phase since its only purpose was to make the nonwords more familiar to the participant prior to the lexical decision part of the experiment.

Apparatus and Materials. The apparatus and materials used in the present experiment were identical to those described in Experiment 1 except for the inclusion of the additional pseudohomophones.

\section{Results}

Mean reaction times and error rates are presented in Table 1 . These data were analyzed in two steps. The first step simply consisted of pairwise $t$ tests to assess whether there was an ambiguity effect on reaction time and error rates in the present experiment. The second step consisted of a $2 \times 2$ mixed ANOVA comparing the ambiguity effects observed in this experiment to those observed in the pseudohomophone condition of Experiment 1.

With respect to the ambiguity effects in the present experiment, both the reaction time and error rate patterns were in the direction consistent with an ambiguity effect. As was the case in Experiment 1, only the error rate difference was statistically significant $[t(14)=3.18, p<.01]$. The $t$ value associated with the reaction time analysis was less than 1.

The critical issue is whether the ambiguity effects observed here were smaller or larger than those observed in the pseudohomophone condition of Experiment 1. When the ambiguity effect in the reaction time data was submitted to a $2 \times 2$ (ambiguity $\times$ experiment) ANOVA, none of the main effects or interactions were significant. However, when an analogous analysis was performed on the error data, there was a significant main effect of ambiguity $\left[F(1,25)=27.00, M S_{\mathrm{e}}=.0014, p<.001\right]$. More important, the interaction between ambiguity and experiment approached significance $\left[F(1,25)=3.43, M S_{\mathrm{c}}=\right.$ $.0014, p=.08]$. Thus, the trend in the data is for the ambiguity effect to increase in magnitude under conditions that further slow the time to identify words correctly.

\section{Discussion}

The results of this experiment are suggestive but again not conclusive. The use of additional nonwords that had been preexposed did increase both the mean reaction time to make correct word decisions and the error rate for those decisions. However, neither of these increases was reliable. The failure to find reliable increases is more due to the large variance that occurred in the present experiment than to a failure to affect word decisions. In fact, correct word reaction times were over $100 \mathrm{msec}$ slower in this experiment and average error rates approximately $4 \%$ higher than in the pseudohomophone condition of Experiment 1 . Thus, despite the lack of statistical significance, the manipulations of the present experiment did have the desired effect of slowing correct word decisions.

The result of this slowing was to slightly reduce the magnitude of the ambiguity advantage relative to that observed in the pseudohomophone condition of Experiment 1 . However, the effect is far from reversed. Thus, our hope of showing both an ambiguity advantage and an ambiguity disadvantage within the same task (i.e., lexical decision) was not fulfilled. Nonetheless, the reduction of the ambiguity effect is consistent enough with the "efficient then inefficient" hypothesis to make further examination of it appear worthwhile.

Given the results of the first two experiments, however, we decided that it may be difficult to demonstrate an ambiguity disadvantage on lexical decision performance. Participants may rely primarily on familiarity when making lexical decisions regardless of the degree to which items are processed, and blend states could have a high degree of familiarity. Thus, it is possible that when participants are in the context of a lexical decision task, they seldom move beyond the blend state. One assumption of the "fast then slow" explanation is that the process of escaping the blend pattern causes an ambiguity disad- 
Table 2

Reaction Times (in Milliseconds) and Percent Error Rates in Experiment 3

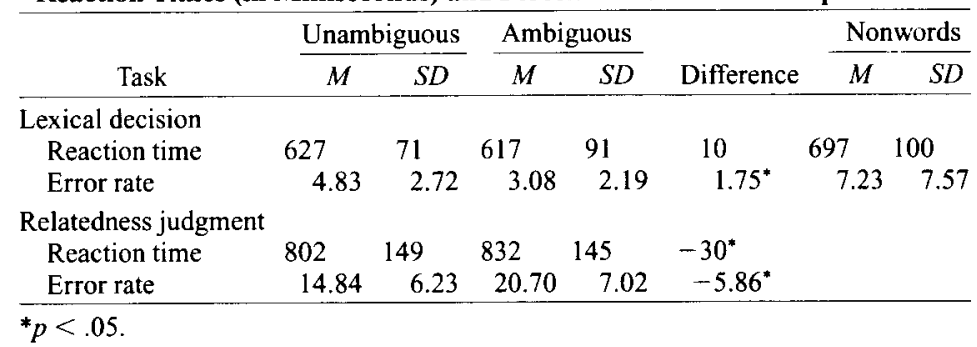

vantage. It may be simply impossible to ever observe such a disadvantage using a task in which blend states are sufficient for accurate responding.

\section{EXPERIMENT 3}

The goal of Experiment 3 was to provide yet a further test of the "efficient then inefficient" explanation of ambiguity effects described throughout the paper. However, in contrast to the previous experiments, we did not attempt to produce an ambiguity disadvantage on lexical decision in the present experiment. Instead, we attempted to show both an ambiguity advantage and an ambiguity disadvantage within the same experiment.

Each trial of the experiment was composed of two responses. First, a stimulus appeared and participants had to make a lexical decision to it. After they responded, a second stimulus appeared that was either related or unrelated to the first. Participants then had to decide whether or not it was related. Reaction time and accuracy were measured for both responses.

The notion behind the experiment is the following. The words presented for the lexical decision portion of the trial are the ambiguous and unambiguous items we have been using throughout. On the basis of the previous experiments, we expect these stimuli to show an ambiguity advantage with respect to lexical decision. However, in order to make the subsequent relation judgment, participants must choose a specific meaning of the first word and, if our theory is correct, this will be harder to do for ambiguous words. Thus, we should see an ambiguity disadvantage for the relation judgments.

\section{Method}

Participants. Fifteen undergraduates from the University of Toronto at Scarborough participated in the experiment. The experiment took approximately $25 \mathrm{~min}$ and participants received one half of a bonus credit toward their introductory psychology class. All participants had normal or corrected vision, and all were sufficiently proficient in English to perform the experiment without difficulty.

Procedure. The experiment was composed of 256 trials. Each trial consisted of the following: (1) a 250-msec blank field, (2) a $500-\mathrm{msec}$ presentation of a plus sign fixation point slightly left of the center of the screen, (3) a 500 -msec blank field, (4) a letter string presented to the left of the fixation point until a lexical decision response was recorded, (5) a 1,000-msec blank screen, (6) a $500-\mathrm{msec}$ presentation of a plus sign fixation point to the right of the center of the screen, (7) a blank 500-msec field, (8) a letter string presented to the right of the fixation point where it remained until a relatedness decision was recorded, and (9) a blank field for $1,000 \mathrm{msec}$ before the next trial began.

When the lexical decision stimulus was a word, the stimulus presented for the relatedness judgment was always a word. On half of the trials it was a word that was related to the first stimulus, and on the other half of the trials it was unrelated. When the first stimulus was a nonword, a nonword was also presented on the relatedness judgment portion of the trial. The purpose of presenting the nonword during the relatedness section of the experiment was to keep the experiment flowing smoothly. The presentation of words and nonwords was randomized throughout the experiment with the constraints that a maximum of four words or nonwords could be presented consecutively and a maximum of four related or unrelated decisions could be presented consecutively.

Participants were instructed to make both their lexical decisions and their relatedness judgments as quickly and accurately as possible. In both cases, the " $z$ " key was used to indicate a "yes" response ("yes it is a word," and "yes it is related"), and the "/" key was used to indicate a "no" response. Participants were informed that when the first stimulus was a nonword, the second stimulus would also be a nonword. They were told that they could press either key for their relatedness decision on such trials.

Apparatus and Materials. The apparatus used in this experiment was identical to that used in the previous experiments. The words presented on the lexical decision portion of each trial were the 64 pairs of ambiguous and unambiguous words used in the previous experiments. The nonwords presented during the lexical decision were the 128 pronounceable nonwords used in Experiment 1.

The words presented in the relatedness portion of the trials were generated by coming up with an item that was either semantically or associatively related to each ambiguous and unambiguous item. In the case of the ambiguous items, we attempted to sometimes choose a word related to what we assumed to be the most dominant meaning and to sometimes choose a meaning related to the second most dominant meaning. Each ambiguous and unambiguous item is presented in the Appendix with its related item.

Once the related stimuli were generated, the pairs of items (i.e., a lexical decision stimulus and its related item) were split into two lists. List A and List B. A reordered version of each list was then generated wherein each lexical decision item was paired with a different lexical decision item's related word, producing unrelated versions of each list (Lists $A^{\prime}$ and $B^{\prime}$ ). Half of the participants then got pairs from Lists $A$ and $B^{\prime}$, whereas the others got items from Lists $A^{\prime}$ and $B$. Thus, across participants, all items occurred in both the related and unrelated conditions.

\section{Results}

The results of the present experiment are presented in Table 2. The lexical decision data reflect the mean reaction time to make a correct decision, and the mean error rate for mistakenly categorizing words as nonwords. The 
relatedness decision data reflect the mean reaction time to make a correct relatedness decision and the mean error rate for incorrectly deciding that two items are related. The means and error rates shown for the relatedness decisions are conditionalized on following a correct lexical decision response.

Lexical decision performance. The lexical decision results are very similar to those observed in the pronounceable nonword condition of Experiment 1. A nonsignificant 10 -msec ambiguity advantage occurred in the reaction time data $(t<1)$. A small but significant $1.75 \%$ ambiguity advantage was observed in the error data $[t(11)=2.43, p<.05]$. Thus, once again, a small ambiguity advantage is apparent in lexical decision performance.

Relatedness judgments. Given the ambiguity advantage observed in the lexical decision portion of each trial, the interesting question is whether there was a subsequent ambiguity disadvantage on the relatedness judgment portion of the trial. Indeed, participants took $30 \mathrm{msec}$ longer to make their relatedness judgments to ambiguous than to unambiguous words, and this difference was significant $[t(11)=2.30, p<.05]$. The participants made $5.86 \%$ more errors when making relatedness judgments to ambiguous words than to unambiguous words. This increase was also statistically significant $[t(11)=5,745$, $p<.05]$. Thus, in contrast to the ambiguity advantage observed in the lexical decisions, an ambiguity disadvantage was observed in the relatedness judgments.

\section{Discussion}

The present experiment was successful in demonstrating both an ambiguity advantage and an ambiguity disadvantage within the same trials. Participants made faster and more accurate lexical decisions to ambiguous words, but slower and less accurate relatedness judgments. This finding is exactly what would be expected if ambiguous words were relatively fast to reach a semantic blend state, but then relatively slow to get out of the blend and get into a learned state.

\section{GENERAL DISCUSSION}

Semantic ambiguity is interesting because it has opposite effects on tasks that should intuitively tap mostly the same processes-lexical decision and reading. We proposed an "efficient then inefficient" explanation that, in concert with assumptions about how the tasks are mapped onto underlying processing, reconciles these seemingly disparate effects. Moreover, this view fits nicely with other findings examining priming effects caused by ambiguous words.

The primary purpose of the experiments reported here was to test our explanation empirically. Our original hope was to demonstrate both an ambiguity advantage and an ambiguity disadvantage within the same task. Although that specific goal was not attained, the results of the pres- ent experiments do support our view. As the time to make correct word decisions was systematically increased via nonword manipulations, the ambiguity effect went from nonexistent, to small, to larger, then back to small. This nonlinear change in ambiguity effects is exactly what is predicted if ambiguous items have a processing advantage early on, but a processing disadvantage later.

We also showed that in a context where participants had to first make a lexical decision to an ambiguous item followed by a relatedness judgment based on that item, the lexical decisions were faster and more accurate for the ambiguous items, whereas the relatedness judgments were slower and less accurate. Presumably this is because lexical decisions can be made relatively early in processing, where ambiguous words have an advantage. Relatedness judgments require a specific meaning for an ambiguous word, which is not obtained until the item has been processed more deeply (i.e., a blend state is not sufficient to support a relatedness judgment).

We see these findings as providing preliminary support for our theoretical framework, a framework that not only makes sense of the ambiguity effects at a more general level, but one that also makes concrete and testable predictions. For example, we are not yet convinced that it is impossible to show both an ambiguity advantage and an ambiguity disadvantage within the same task, though we doubt whether this could be done using the lexical decision task. An interesting possibility would be to come at this issue from the other side. That is, if the typical reading paradigm could be modified in a manner that made the processing of specific meanings unnecessary, would the ambiguity disadvantage typically seen in that task turn into an advantage? Said another way, an attempt to turn a disadvantage into an advantage may turn out to be the more successful strategy after all.

\section{REFERENCES}

BeSNer, D., \& JoORdens, S. (1995). Wrestling with ambiguity-Further reflections: Reply to Masson and Borowsky (1995) and Rueckl (1995). Journal of Experimental Psychology: Learning. Memory, \& Cognition, 21, 515-519.

BorowsKy, R., \& MASSON, M. E. J. (1996). Semantic ambiguity effects in word identification. Journal of Experimental Psychology: Learning, Memory, \& Cognition, 22, 63-85.

DufFY, S. A., Morris, R. K., \& RAYNER, K. (1988). Lexical ambiguity and fixation times in reading. Journal of Memory \& Language, 27, 429-466.

GERNSBACHER, M. A. (1984). Resolving 20 years of inconsistent interactions between lexical familiarity and orthography, concreteness, and polysemy. Journal of Experimental Psychology: General, 113, 256-280.

JAMES, C. T. (1975). The role of semantic information in lexical decisions. Journal of Experimental Psychology: Human Perception \& Performance, 1, 130-136.

JASTRZEMBSKI, J. E. (1981). Multiple meanings, number of related meanings, frequency of occurrence, and the lexicon. Cognitive Psychology, 13, 278-305

JastrzembSKi, J. E., \& Stanners, R. F. (1975). Multiple word meanings and lexical search speed. Journal of Verbal Learning \& Verbal Behavior, 14, 534-537. 
JoORdENS, S., \& BECKER, S. (1997). The long and short of semantic priming effects in lexical decision. Journal of Experimental Psychology: Learning, Memory, \& Cognition, 5, 1083-1105.

JoORdENS, S., \& BESNER, D. (1994). When banking on meaning is not (yet) money in the bank: Explorations in connectionist modeling. Journal of Experimental Psychology: Learning, Memory, \& Cognition, 20, 1051-1062.

JOORDENS, S., \& HOCKLEY, W. E. (in press). Recollection and familiarity through the looking glass: When old does not mirror new. Journal of Experimental Psychology: Learning, Memory, \& Cognition.

Joordens, S., \& Piercey, C. D. (1996, November). The dynamic nature of lexical decision: Implications for models of memory. Poster presented at the 37th Annual Meeting of the Psychonomic Society, Chicago.

Kellas, G., Ferraro, F. R., \& Simpson, G. B. (1988). Lexical ambiguity and the time-course of attentional allocation in word recognition. Journal of Experimental Psychology: Human Perception \& Performance, 14, 601-609.

MARCEL, A. J. (1980). Conscious and preconscious recognition of polysemous words: Locating the selective effects of prior verbal context. In R. S. Nickerson (Ed.), Attention and performance VIII (pp. 435457). Hillsdale, NJ: Erlbaum

Masson, M. E. J. (1991). A distributed memory model of context effects in word identification. In D. Besner \& G. W. Humphreys (Eds.), Basic processes in reading: Visual word recognition (pp. 233-263). Hillsdale, NJ: Erlbaum.

MASSON, M. E. J., \& BoRowsky, R. (1995). Unsettling questions about semantic ambiguity in connectionist models: Comment on Joordens and Besner (1994). Journal of Experimental Psychology: Learning. Memory, \& Cognition, 21, 509-514.

Millis, M. L., \& ButTons, S. B. (1989). The effect of polysemy on lexical decision time: Now you see it, now you don't. Memory \& Cognition, 4, 141-147.

ONIFER, W., \& SWINNEY, D. A. (1981). Accessing lexical ambiguities during sentence comprehension: Effects of frequency of meaning and contextual bias. Memory \& Cognition, 9, 225-236.

RAYNER, K., \& DUFFY, S. A. (1986). Lexical complexity and fixation times in reading: Effects of word frequency, verb complexity, and lexical ambiguity. Memory \& Cognition, 14, 191-201.

RAYNER, K., \& DUFFY, S. A. (1987). Eye movements and lexical ambigu- ity. In J. K. O'Regan \& A. Levy-Schoen (Eds.), Eye movements: From physiology to cognition (pp. 521-529). Amsterdam: North-Holland. RaYNer, K., \& Frazier, L. (1989). Selection mechanisms in reading lexically ambiguous words. Journal of Experimental Psychology: Learning, Memory, \& Cognition, 15, 779-790.

RUECKL, J. G. (1995). Ambiguity and connectionist networks: Still settling into a solution-Comment on Joordens and Besner (1994). Journal of Experimental Psychology: Learning, Memory, \& Cognition, 21, 509-514

Seidenberg, M. S., Tanenhaus, M. K., Leiman, J. M., \& Bienkowski, M. (1982). Automatic access of the meanings of ambiguous words in context: Some limitations of knowledge toward processing. Cognitive Psychology, 14, 489-537.

Stone, G. O., \& VAN ORden, G. C. (1993). Strategic control of processing in word recognition. Journal of Experimental Psychology: Human Perception \& Performance, 19, 744-774.

SWINNEY, D. A. (1979). Lexical access during sentence comprehension: (Re)consideration of context effects. Journal of Verbal Learning \& Verbal Behavior, 18, 645-659.

\section{NOTES}

1. In fact, Masson (1991) reported a simulation of Seidenberg et al.'s (1982) experiment in which he found that ambiguous related items primed targets as well as unambiguous related items if the target was presented immediately after the prime, but not as well when there was a delay between the prime and target. This result is also viewed as supporting the notion that both meanings of an unambiguous word are available early in processing, but only one is available later. The fact that Masson was able to simulate these results adds further support to the contention that the processing characteristics of an attractor network may provide a good framework for conceptualizing differences between ambiguous and unambiguous words.

2. Only participant analyses are reported here because we did not code the appropriate information to perform item analyses. Although this does not compromise the reliability of our results at all, the generality of our findings to other stimulus sets would have been further strengthened had we been able to show that the item analyses produced results paralleling the participant analyses. 
APPENDIX

Related Items Used in Experiment 3

\begin{tabular}{|c|c|c|c|c|c|}
\hline ate & food & ace & card & bet & gamble \\
\hline bat & vampire & bloat & stomach & bluff & cliff \\
\hline bite & teeth & bolt & lightening & buzz & bee \\
\hline boot & kick & bend & twist & bowl & fruit \\
\hline bold & brave & buck & bronco & chance & luck \\
\hline charge & fee & chain & gang & chest & treasure \\
\hline curve & $\operatorname{arch}$ & coast & ocean & duke & baron \\
\hline dash & hyphen & deep & dark & date & month \\
\hline disk & frisbee & deck & wood & drain & $\operatorname{clog}$ \\
\hline draft & beer & dish & soap & drag & pull \\
\hline dusk & dawn & duck & goose & force & demand \\
\hline field & baseball & fear & anger & fine & small \\
\hline fame & fortune & foil & $\operatorname{tin}$ & grown & mature \\
\hline grade & slope & grain & wheat & grave & tomb \\
\hline hunt & kill & hail & rain & hug & embrace \\
\hline hog & greedy & hitch & trailer & hound & dog \\
\hline jolt & shock & jerk & goof & jump & leap \\
\hline joint & elbow & love & heart & land & dirt \\
\hline loud & noise & lean & trim & large & big \\
\hline light & feather & lint & pocket & loaf & bread \\
\hline meat & burger & match & same & maze & labyrinth \\
\hline $\operatorname{mint}$ & money & mind & brain & miss & skip \\
\hline plug & socket & palm & hand & pig & swine \\
\hline pet & stroke & pond & water & pound & weight \\
\hline plumb & level & punch & hit & rum & rye \\
\hline ram & sheep & rain & storm & rock & music \\
\hline rice & pasta & roll & turn & rev & engine \\
\hline row & column & sane & rational & sack & fire \\
\hline stream & brook & screen & door & soup & stew \\
\hline seal & envelope & shoe & foot & shed & remove \\
\hline soap & clean & $\sin k$ & faucet & steak & barbecue \\
\hline spade & shovel & spent & exhausted & spring & boing \\
\hline steam & boil & stall & horse & stuck & stranded \\
\hline star & movie & stack & pile & steer & bull \\
\hline stuff & things & stick & branch & strife & hardship \\
\hline strike & picket & storm & cloud & strip & undress \\
\hline sworn & pledge & swamp & marsh & swept & broom \\
\hline switch & swap & tee & golf & $\operatorname{tag}$ & price \\
\hline turf & grass & tick & flea & tea & coffee \\
\hline tip & waiter & tent & camp & tire & exhausted \\
\hline town & city & type & font & valve & intake \\
\hline vault & leap & wash & shampoo & wake & funeral \\
\hline worth & value & watch & clock & & \\
\hline
\end{tabular}

(Manuscript received September 8, 1997;

revision accepted for publication June 8,1999 .) 